\title{
Granular Computing: Past, Present and Future
}

\author{
Yiyu Yao \\ Department of Computer Science, University of Regina \\ Regina, Saskatchewan, Canada S4S 0A2 \\ Email: yyao@cs.uregina.ca URL: http://www.cs.uregina.ca/ yyao
}

\begin{abstract}
Granular computing is gradually changing from a label to a new field of study. The driving forces, the major schools of thought, and the future research directions on granular computing are examined. A triarchic theory of granular computing is outlined. Granular computing is viewed as an interdisciplinary study of human-inspired computing, characterized by structured thinking, structured problem solving, and structured information processing.
\end{abstract}

\section{Introduction}

To begin the Forward to Knowledge Space: Theories, Empirical Research, and Applications [1], Falmagne makes the following insightful comment: "A scientific field in its infancy - if it is destined for maturity - requires loving care in the form of a steady diet of fresh ideas and results." While recognizing, in a very slight sense, the role of fate and luck, Falmagne emphasizes the importance of novel ideas and results that foster a healthy growth of a new scientific field. Pondering on this remark, I asked myself two questions. What are the ingredients of a balanced, steady diet for granular computing? How do we provide it? The answers may be sought from both a historical and a futuristic perspective.

This paper contains ideas from my many attempts to (partially) answer these two questions, which offers a very optimistic view on the future of granular computing. By exploring a different avenue, J.T. Yao attempts to answer the same questions through a systematic citation analysis of the published works on granular computing [31, 32]. The results shed a different light on the past, present and future of granular computing research and provide some evidence supporting my arguments.

The three parts of the paper are devoted to the past, present and future of granular computing, respectively. In Section 2, I present arguments to support an optimistic view of granular computing, as a new paradigm of humaninspired computing and problem solving, by drawing results from studies on intelligence and systems science. In Section 3, I outline a triarchic theory of granular computing and present an overview of artificial intelligence related research on granular computing. In Section 4, I discuss a few issues related to the future of granular computing.

\section{The Rise of Granular Computing}

Several authors discussed the roots of granular computing in terms of earlier developments in mathematics [3]. I will restrict the discussion to more recent developments of granular computing that have helped transform it from a label to a field of study.

\subsection{An emerging field}

It is well accepted that the theories of fuzzy sets and rough sets are two main contributors to the emergence of granular computing $[2,11,17,24,27,34]$. A citation analysis of published works shows that the top 15 highly cited papers of granular computing before 2007 are all related to fuzzy sets and rough sets [31]. In the context of fuzzy sets, the term "information granulation" was suggested by Zadeh in 1979 [42]. However, the movement of granular computing did not start until 1997 when his seminal paper on the same topic was published, in which the term "granular computing" is explicitly used $[31,43]$. The theory of rough sets started in early 1980's [25]. Although the notion of "granularity" is not explicitly used by researchers, it indeed plays a fundamental role in rough set theory [27]. In retrospect, it is fair to say that rough set theory, as both a vigorous theory and a concrete example of granular computing, brought on the rise of granular computing [39].

Granular computing started as "a label of theories, methodologies, techniques, and tools that make use of granules in the process of problem solving" [33]. With more than a decade of study, we begin to see the convergence towards a field of study. It seems that a common theme is converging to a view that granular computing is an interdisciplinary study of computations in nature, society and sci- 
ence, with an underlying notion of multiple levels of granularity [41]. It represents a shift from machine-centric computing towards nature- and human-inspired computing.

As a multidisciplinary and cross-disciplinary study, granular computing draws results from many fields and integrates these results. Granular computing deals with both humans and machines. The ideas and principles of granular computing may be extracted from human problem solving, computing and information processing. They in turn can be applied to design and implement human-inspired machines and algorithms for problem solving, computing and information processing.

\subsection{An optimistic view}

I will now elaborate on two more reasons for my optimistic view on the human-inspired paradigm of granular computing [40].

\subsubsection{Moravec's paradox}

In the field of artificial intelligence, Moravec's paradox [22] was articulated more than 20 years ago. It represents the dichotomy between machines and humans regarding the complexity or easiness in solving different problems. Contrary to what researchers first assumed and believed, machines can do well things that humans find hard, and perform poorly on what is seemingly effortless for humans.

A plausible explanation for the Moravec's paradox is based on the theory of evolution [22]. The human brain and all human skills are results of Darwinian evolution and they are implemented biologically. This natural selection process gradually and continually improves and optimizes such biological designs and implementations. Older skills, such as recognizing faces, recognizing voices, moving around in space, etc., are fully evolved and mastered by humans. We can perform these perception-based tasks almost unconsciously and they therefore appear to us to be effortless. For the same reason, reengineering the working principles underlying the human brain and older human skills are much more difficult, as there is a lack of precisely specified procedures.

Abstract thinking is a new trick developed more recently in human evolutionary history and one we have not fully mastered yet. It therefore seems intrinsically difficult when we do it, as we must follow some precisely defined and constructed procedures. Such skills may not necessarily be difficult to reengineer and thus machines may easily duplicate them.

Many authors attribute the lack of breakthroughs in artificial intelligence to our limited understanding of the human brain and human ways of problem solving [9,23]. Recently, there is a new surge of research interest in understanding the human brain and natural intelligence [4, 9, 21, 23, 45].
Two well cited sources that motivate the study of granular computing are Hobbs' paper on a theory of granularity [10] and Zadeh's paper on fuzzy information granulation [43]. Hobbs [10] argues that we look at the world under various grain sizes, and furthermore, we abstract only those things that serve our present interests. He writes[10],

Our ability to conceptualize the world at different granularities and to switch among these granularities is fundamental to our intelligence and flexibility. It enables us to map the complexities of the world around us into simple theories that are computationally tractable to reason in. If we are to have a machine of even moderate intelligence, it must have a theory of granularity woven into the very foundation of its reasoning processes.

Zadeh [43] describes the human cognition and its relevance to granular computing as follows:

Among the basic concepts which underlie human cognition there are three that stand out in importance. The three are: granulation, organization and causation. In a broad sense, granulation involves decomposition of whole into parts; organization involves integration of parts into whole; and causation relates to association of causes with effects.

Inspired by the ways in which humans granulate human concepts we can proceed to granulate conceptual structures in various fields of science.

These two papers greatly influenced my view of granular computing, and perhaps those of many others [13, 44].

In explaining human intelligence and human problem solving, Minsky [20] discusses several important features. Humans have many "Ways to Think" and can also create new "Ways to Think." Humans form multiple representations of the world. Minsky portrays a typical brain as containing a great many different parts called "resources." The concept of "resources" is used as an abstract notion without a direct linkage to biological implementation in a brain. A state of mind is explained from the activities of a certain collection of mental resources.

In a previous paper [40], I tie all these ideas together and argue that granular computing may be viewed as a particular class of "Ways to Think." It emphasizes on the effective use of multiple levels of granularity. The notion of "resources" may be related to the notion of "granules." This integration of ideas immediately offers a conceptualization of granular computing as human-inspired computing.

\subsubsection{Systems theory}

In the same paper [40], I compare the recent rise of granular computing with the rise of systems theory a few decades 
earlier, in terms of their philosophies, goals, scopes, and methodology.

The general systems theory attempts to discover and investigate structures and underlying principles common to most natural and artificial systems [5, 16, 28, 29]. It is motivated and developed based on an observation that similar general conceptions and viewpoints have been evolved independently in various branches of science. A hierarchy with many levels is used as a central notion to describe the structural similarities, structural uniformities and logical homologies of many different systems. Thus, the general systems theory is viewed as a science of sciences in an attempt to arrive at unity through diversity [8], namely, the "Unity of Science" [29].

Research of granular computing attempts to discover and investigate structures and underlying principles common to most types of human problem solving $[39,40]$. As such, granular computing may be viewed as both an art and a science of human-inspired computing and problem solving. To build a theory of granular computing, it is necessary to extract high-level commonalities of different disciplines and to synthesize their results into an integrated whole by ignoring low-level details. It is also necessary to make explicit ideas hidden in discipline-specific discussions in order to arrive at a set of discipline-independent principles [39].

In granular computing, a hierarchy is also used as a central notion $[34,35,36,37,38,39,40]$ to describe, represent and interpret our multilevel perception and understanding of reality. This unified language of granular computing, as human-inspired computing, is thus based on integrative multiple levels of granularity. The study of a general theory of granularity is perhaps a main task of granular computing $[12,13]$.

Searching for unity from diversity motivates researchers of granular computing in a similar way as it motivated researchers of systems science. The study of system science have led to a great progress towards the "Unity of Science." The current study of granular computing will bring us closer to a new paradigm of human-inspired computing. The implication of this paradigm for the future generation of cognitive systems, including also human-machine integrated systems, is very significant.

\section{Current Research on Granular Computing}

In this section, I will outline a triarchic theory of granular computing, consisting of the philosophical, methodological and computational perspectives. It is possible to review and compare current research results from the three perspectives. However, since research related to fuzzy sets and rough sets has been well documented [2, 11, 17, 24, 27, $31,32,34]$, I will focus only on artificial intelligence related research.

\subsection{A triarchic theory of granular computing}

The triarchic theory consists mainly of granular structures and the granular computing triangle [39].

\subsubsection{Granules and granular structures}

A primitive notion of granular computing is a granule representing a part of a whole. Granules are the focal points of our current interest or the units we used to obtain a description or a representation. A granule plays two distinctive roles. It may be an element of another granule and is considered to be a part forming the other granule. It may also consist of a family of granules and is considered to be a whole. Its particular role is determined by our focal points at different stages of problem solving.

We may collect a family of granules of a similar type together and study their collective properties. This leads to the notion of levels. A level is populated by granules of the similar size or nature. While each granule provides a local view, a level provides a global view.

An important property of granules and levels is their granularity. The granularity of granules and levels enables us to construct a hierarchical structure called a hierarchy. The term hierarchy is used to denote a family of partially ordered levels, in which each level consists of a family of interacting and interrelated granules.

While a single hierarchy offers one representation and understanding with many levels of granularity, multiple hierarchies afford a complete understanding from many views. Granular structures are therefore a family of hierarchies.

\subsubsection{The granular computing triangle}

With granular structures, granular computing emhpasizes on structuredness and structured ways. The granular computing triangle summarizes pictorially the three mutually supporting perspectives on viewing, constructing and working with granular structures. Each vertex represents a specific perspective. The three perspectives are [39]:

Philosophy: structured thinking. It combines analytical thinking for decomposing a whole into parts and synthetical thinking for integrating parts into a whole.

Methodology: structured problem solving. It promotes systematic approaches, effective principles, and practical heuristics and strategies for solving real-world problems. Three tasks are: constructing granular structures, working within a particular level of the structure, and switching between levels.

Computation: structured information processing. Computation is modeled based on two related basic notions: representation and process. Representation covers the formal 
and precise description of granules and granular structures. Processes deal with procedures of granulation and computation with granules.

The three perspectives of granular computing are connected and mutually support each other. Their integration puts granular computing research on a firm basis. In addition, the granular computing triangle recommends a research direction towards an interdisciplinary wholeness approach, rather than scattered, isolated studies.

\subsection{Natural and artificial intelligence}

Problem solving relies on both a repository of knowledge and the intelligence and wisdom required for using such knowledge. An effective strategy developed by humans is known as abstraction or thinking at multiple levels of abstraction $[15,30]$. This establishes a connection between natural and artificial intelligence and granular computing.

\subsubsection{Conceptual modeling of the brain}

A key to understanding natural intelligence is to study the working mechanism of the brain. Several conceptual models of the brain provide evidence supporting granular computing.

A conceptual model of the mind has been proposed in evolutionary psychology [7]. The mind is viewed as a set of special purpose thinking devices or computational modules. The massively modular architecture may be viewed as the result of natural selection; each module is adaptively tailored to solve a particular problem confronted by our ancestors. Minsky [20] suggests a similar simple and elegant model of the brain containing a great many different parts called "resources." A resource may be viewed as a computational module.

Hawkins [9] uses the notion of a cortical hierarchy for deriving a memory-prediction framework for explaining intelligence. In this top-down approach for understanding the brain, a conceptual model of the cortex is given by highlighting its hierarchical connectivity and information flows up and down the hierarchy. According to Hawkins, "the cortex's hierarchical structure stores a model of the hierarchical structure of the real world. The real world's nested structure is mirrored by the nested structure of our cortex."

One may interpret computational modules, resources, and cortex regions as granules. These conceptual models may help us to explain intelligence in terms of multiple levels of granularity.

\subsubsection{Granular logics}

Logic is a formal and precise language for abstract thinking and reasoning. Logical approaches to granular computing take several forms $[10,14,18,19,46]$.
Logical approaches to granular computing are closely related to the classical view of concepts. A concept is defined jointly by the common, intrinsic properties that are applicable to the objects of the concept and a set of objects that are instances of the concept. They are called the intension and the extension of the concept, respectively. With a logic language, a formula is treated as a description of a granule (i.e., the intension of a concept) and the granule itself is a set of objects forming the granule (i.e., the extension of a concept). This allows us to study granular computing in both a logical setting and a set-theoretical setting [46].

A logic language can be used to describe the equivalence of objects, which in turn can be used to form a granule of objects that are indiscernible based on the language. This approach is used by Hobbs [10] for developing a theory of granularity [13] and by Pawlak [26] for eveloping the rough set theory. Liu $[18,19]$ and colleagues extend the strict logic equivalence into a weak equivalence, through which similar objects can be grouped together.

A crucial issue of the logical approaches is the determination of a set of atomic formulas that defines a family of elementary granules, from which a multilevel granular structure can be derived. A detailed description of this issue can be found in [46].

\subsubsection{Problem space and search}

A systematic and automatic problem solving method developed in artificial intelligence is problem space, or state space, search. Hierarchical problem solving is an excellent example that explores multiple levels of abstraction and granularity [14, 44].

Knoblock [14] reviews a number of studies on hierarchical problem solving and proposes an automatic method of generating abstractions. Broadly, hierarchical problem solvers explore one or more abstractions of a problem space. This will significantly reduce the search in the problem solving process, as an abstract space normally contains a much smaller number of states. In order to guarantee that a hierarchical problem solver produces a correct solution, it is necessary to consider some invariant properties. For example, the monotonicity property states that the "existence of ground-level solution implies the existence of an an abstract-level solution," and furthermore, an abstract-level solution can be refined into a ground-level solution. While the first part focuses on the existence of a solution, the second part requires that the solution can be constructed.

Zhang and Zhang [44] propose a quotient space theory of problem solving. The basic idea is to granulate a problem space by considering relationships between states. Similar states are grouped together at a higher level. This produces a hierarchical description and representation of a problem. Like other theories of hierarchical problem solving, quo- 
tient space theory searches a solution using multiple levels, from abstract-level spaces to the ground-level space. Again, invariant properties are considered, including truth preservation downwards in the hierarchy and falsity preservation upwards in the hierarchy. The truth-preservation property is similar to the monotonicity property.

These hierarchical problem solving methods may be viewed as structured problem solving. Structured programming, characterized by top-down design and step-wise refinement based on multiple levels of details, is another example. The principles employed may serve as a methodological basis of granular computing.

\section{Future Research and Concluding Remarks}

Granular computing, as an interdisciplinary and a multidisciplinary study, concerns human-inspried computing and problem solving. The triarchic theory enables us to study granular computing as a field in its wholeness [39, 40, 41]. It draws results from many fields: 1) Results from the general systems theory, regarding efficient evolution of complex systems and organized complexity, suggest that multilevel hierarchical granular structures used in granular computing are suitable for describing complex real-world problems. 2) Results from cognitive science and psychology on human guessing, knowing, thinking and languages provide evidence to support the philosophical view of granular computing. 3) The systematic study of human problem solving, strategies and heuristics, knowledge representation and search, in artificial intelligence and computer programming may provide the necessary models, methods, and tools, which serves as a methodological foundation of granular computing. 4) Advances in information processing and related systems may help us to establish a computational basis of granular computing.

J.T. Yao's [32] study recommends two intermediate tasks for researchers in granular computing. We need to unify terms used in granular computing for easier communication of ideas. It is the time that we interact and communicate among ourselves and with researchers in other communities. These two tasks, according to Crane's [6] study, are crucial to the growth of a scientific field.

A steady diet for granular computing may be provided in several ways. 1) We need to emphasize a balanced approach by considering all three perspectives. 2) We need to stress diversity in order to arrive at a unity. 3) We need to cross the boundaries of different disciplines in order to observe their commonalities and to use them for granular computing. 4) We need to shift from machine-oriented approaches to human-inspired approaches by studying the human brain and natural intelligence first. 5) We need to move beyond the current rough sets and fuzzy sets dominated research agenda in order to make granular computing a more general theory of problem sovling. 6) We need to learn to appreciate both the ideas and concrete results. At the present stage, granular computing is more about an attractive idea than a fully-grown theory. We must give it loving care to ensure a wonderful blossom.

\section{References}

[1] Albert, D. and Lukas, J. (Eds.) Knowledge Space: Theories, Empirical Research, and Applications, Mahwah, New Jersey: Lawrence Erlbaum Associates, Publishers, 1990.

[2] Bargiela, A. and Pedrycz, W. Granular Computing: An Introduction, Boston: Kluwer Academic Publishers, 2002.

[3] Bargiela, A. and Pedrycz, W. The roots of granular computing, Proceedings of 2006 IEEE International Conference on Granular Computing, 806-809, 2006.

[4] Berwick, R.C., Knight, T.F., Shrobe, Jr. H.E., Sussman, G.J., Ullman, S., Winston, P.H. and Yip, K. The Explanation of Human Intelligence: A Roadmap for Research, http://groups.csail.mit.edu/genesis/HIE/ white.html (accessed March 2, 2008).

[5] Capra, F. The Web of Life, New York: Anchor Books, 1997.

[6] Crane, D. Invisible Colleges: Diffusion of Knowledge in Scientific Communities, Chicago: The University of Chicago Press, 1972.

[7] Downes, S.M. Evolutionary psychology, Stanford Encyclopedia of Philosophy, http://plato.stanford.edu/entries/evolutionarypsychology/ (accessed March 9, 2008).

[8] Gray, W. and Rizzo, N.D. (Eds.) (1973) Unity Through Diversity, I, II, New York: Gordon and Breach Science Publishers, 1973.

[9] Hawkins, J. (with Blakeslee, S.) On Intelligence, New York: Henry Holt and Company, 2004.

[10] Hobbs, J.R. Granularity, Proceedings of the Ninth International Joint Conference on Artificial Intelligence, 432-435, 1985.

[11] Inuiguchi, M., Hirano, S. and Tsumoto, S. (Eds.) Rough Set Theory and Granular Computing, Springer, Berlin, 2003.

[12] Keet, C.M. A taxonomy of types of granularity, Proceeding of 2006 IEEE International Conference on Granular Computing, 106-111, 2006.

[13] Keet, C.M. A Formal Theory of Granularity, PhD Thesis, Faculty of Computer Science, Free University of Bozen-Bolzano, Italy, http://www.meteck.org/files/ AFormalTheoryOfGranularity_CMK08.pdf (accessed June 8, 2008). 
[14] Knoblock, C.A. Generating Abstraction Hierarchies: An Automated Approach to Reducing Search in Planning, Boston: Kluwer Academic Publishers, 1993.

[15] Kramer, J. Is abstraction the key to computing? Communications of the ACM, 50, 36-42, 2007.

[16] Laszlo, E. The Systems View of the World: The Natural Philosophy of the New Developments in the Science, New York: George Brasiller, 1972.

[17] Lin, T.Y., Yao, Y.Y. and Zadeh, L.A. (Eds.) Data Mining, Rough Sets and Granular Computing, Heidelberg: Physica-Verlag, 2002.

[18] Liu, Q. and Sun, H. Theoretical study of granular computing, Proceeding of RSKT 2006, LNAI 4062, 93102, 2006.

[19] Liu, Q. and Wang, Q. Granular logic with closeness relation $\sim_{\lambda}$ and its reasoning, Proceeding of RSFDGrC 2005, LNAI 3641, 709-717, 2005.

[20] Minsky, M. The Emotion Machine: Commonsense Thinking, Artificial Intelligence, and the Future of the Human Mind, New York: Simon \& Schuster Paperbacks, 2007.

[21] Mitchell, T. AI and the Impending Revolution in Brain Sciences, http://www.cs.cmu.edu/\%7Etom/pubs/ AAAI-PresAddr.pdf (accessed March 1, 2008).

[22] Moravec, H. Mind Children: the Future of Robot and Human Intelligence, Cambridge: Harvard Unversity Press, 1988.

[23] Morris, R., Tarassenko, L. and Kenward, M. (Eds.) Cognitive Systems: Information Processing Meets Brain Science, New York: Elsevier, 2006.

[24] Nguyen, H.S., Skowron, A., and Stepaniuk, J. Granular computing: a rough set approach, Computational Intelligence, 17, 514-544, 2001.

[25] Pawlak, Z. Rough sets, International Journal of Computer and Information Sciences, 11, 341-356, 1982.

[26] Pawlak, Z. Rough Sets, Theoretical Aspects of Reasoning about Data, Dordrecht: Kluwer Academic Publishers, 1991.

[27] Pawlak, Z. Granularity of knowledge, indiscernibility and rough sets, Proceedings of the IEEE International Conference on Fuzzy Systems, 106-110, 1998.

[28] Skyttner, L. General Systems Theory, Ideas \& Applications, Singapore: World Scientific, 2001.

[29] von Bertalanffy, L. An outline of general system theory, The British Journal for the Phi-losophy of Science, 1, 134-165, 1950.

[30] Wing, J.M. Computational thinking, Communications of the ACM, 49, 33-35, 2006.
[31] Yao, J.T. A ten-year review of granular computing, Proceedings of 2007 IEEE International Conference on Granular Computing, 734-739, 2007.

[32] Yao, J.T. Recent developments of granular computing, Proceedings of 2008 IEEE International Conference on Granular Computing, 2008.

[33] Yao, Y.Y. Granular computing: basic issues and possible solutions, Proceedings of the 5th Joint Conference on Information Sciences, 186-189, 2000.

[34] Yao, Y.Y. Information granulation and rough set approximation, International Journal of Intelligent Systems, 16, 87-104, 2001.

[35] Yao, Y.Y. A partition model of granular computing, LNCS Transactions on Rough Sets, 1, LNCS , 232-253, 2004.

[36] Yao, Y.Y. Granular computing, Computer Science (Ji Suan Ji Ке Хие), 31, 1-5, 2004.

[37] Yao, Y.Y. Perspectives of granular computing, Proceedings of 2005 IEEE International Conference on granular computing, Vol. 1, 85-90, 2005.

[38] Yao, Y.Y. Three perspectives of granular computing, Journal of Nanchang Institute of Technology, 25, 1621, 2006.

[39] Yao, Y.Y. The art of granular computing, LNAI 4585, 101-112, 2007.

[40] Yao, Y.Y. The rise of granular computing, Journal of Chongqing University of Posts and Telecommunications (Natural Science Edition), 20, 299-308, 2008.

[41] Yao, Y.Y. Granular computing: past, present, and future, Proceedings of RSKT'08, LNAI 5009, 27-28, 2008.

[42] Zadeh, L.A. Fuzzy sets and information granularity, in: Advances in Fuzzy Set Theory and Applications, Gupta, N., Ragade, R. and Yager, R. (Eds.), Amsterdam: North-Holland, 3-18, 1979.

[43] Zadeh, L.A. Towards a theory of fuzzy information granulation and its centrality in human reasoning and fuzzy logic, Fuzzy Sets and Systems, 19, 111-127, 1997.

[44] Zhang, L. and Zhang, B. Theory and Application of Problem Solving: Theory and Application of Granular Computing in Quotient Spaces (in Chinese), 2nd edition, Beijing: Tsinghua University Press, 2007.

[45] Zhong, N., Liu, J., Yao, Y.Y., Wu, J., Lu, S. and Li, K. (Eds.) Web Intelligence Meets Brain Informatics, LNAI 4845, Berlin: Springer, 2007.

[46] Zhou, B. and Yao, Y.Y. A logic approach to granular computing, International Journal of Cognitive Informatics and Natural Intelligence, 2, 63-79, 2008. 